\title{
碘促进吡喃并[3,2-c]喹啉-4,5-二酮类衍生物的有效合成
}

\author{
周婷王道林* 钱建华 赵伟 \\ (渤海大学化学化工学院 辽宁省功能化合物合成与应用重点实验室＼cjkstart锦州 121003)
}

\begin{abstract}
摘要 在超声波辅助及哌啶和聚乙二醇-400 的协同作用下，通过 1-甲基-3-乙酰基-4-羟基-2-喹啉酮与芳香醛的 Aldol 缩 合反应，合成了 1-甲基-3-肉桂酰基-4-羟基-2-喹啉酮. 该缩合产物在 $\mathrm{I}_{2}$ 促进下，经过分子内的环化反应，成功制备了一 系列吡喃并[3,2-c]喹啉-4,5-二酮衍生物. 该方法具有反应操作简单、条件温和、收率良好的特点.
\end{abstract}

关键词＼cjkstart喹啉; 杂环; 超声波; 碘; 合成

\section{Iodine Promoted Synthesis of Pyrano[3,2-c]quinoline-4,5-diones}

\author{
Zhou, Ting Wang, Daolin* Qian, Jianhua Zhao, Wei \\ (Key Laboratory of Synthesis \& Application of Functional Compound, College of Chemistry and \\ Chemical Engineering, Bohai University, Jinzhou 121003)
}

\begin{abstract}
An efficient method for the synthesis of pyrano[3,2-c]quinoline-4,5-dione derivatives via iodine promoted intramolecular cyclization of 1-methyl-3-cinnamoyl-4-hydroxyquinolin-2-one, synthesized from aldol condensation of 1-methyl-3-acetyl-4-hydroxyquinolin-2-one with aromatic aldehydes in the presence of pyridine and PEG-400 under ultrasound irradiation, has been described. This method offers the advantages of a green approach, easy work-up, mild conditions and good yields.
\end{abstract}

Keywords quinoline; heterocycle; ultrasound irradiation; iodine; synthesis

吡喃并 [3,2-c]喹啉酮来源于 Rutaceae 植物家族中的 生物碱, 它的许多衍生物具有广泛的生物活性, 如抗凝 血、抗肿瘤、抗高血压、抗炎和抗疮活性 ${ }^{[1]}$. 其中一些 衍生物显示出对癌细胞生长的抑制活性, 被认为是潜在 的抗癌药物 ${ }^{[2]}$. 在有机合成上被用于制备其他天然产物, 如二聚喹啉生物碱和多环杂环化合物等 ${ }^{[3]}$.

吡喃并 [3,2-c]喹啉酮骨架结构由喹啉酮和吡喃酮拼 接组成. 喹啉酮衍生物已发现具有抗症 ${ }^{[4]}$ 、抗增殖 ${ }^{[5]}$ 、抗 乙型肝炎病毒 ${ }^{[6]}$ 和抗 $\mathrm{HIV}^{[7]}$ 活性. 吡喃酮类化合物由于 良好的抗菌 ${ }^{[8]}$ 、抗肿瘤 ${ }^{[9]}$ 等生物活性和药理活性, 在临床 医学上得以应用.

在过去的几十年里, 由于分子碘的无毒、廉价和环 境友好等特性, 使其成为一种应用广泛的有机催化 剂 ${ }^{[10]}$, 用于有效促进化学反应 ${ }^{[1]}$, 如 Michael 加成 ${ }^{[12]}$ 、 催化氧化 ${ }^{[13]}$ 等. 分子碘在有机合成中的重要作用, 已经 愈来愈受到人们的关注 ${ }^{[14]}$.

近年来, 我们以喹啉类杂环化合物为研究对象, 合
成了具有潜在生物活性的含有呋喃 ${ }^{[15 a]}$ 、噻吩 ${ }^{[15 b]}$ 、嘧 啶 ${ }^{[15 c]}$ 和香豆素 ${ }^{[15 d]}$ 等结构单元的喹啉类稠杂环化合物. 为进一步开展此领域的研究工作, 本文以 1-甲基-3-乙 酰基-4-差基-2-喹啉酮为反应底物，在碘的促进下，对 吡喃并[3,2-c]喹啉-4,5-二酮类衍生物的合成方法进行探 讨, 合成路线如 Scheme 1 所示.

\section{1 结果与讨论}

\subsection{3 -肉桂酰基-4-羟基-2-喹啉酮的合成}

有关 3-肉桂酰基-4-羊基-2-喹啉酮的制备, 文献报 道了在 $L$-脯氨酸催化下 ${ }^{[16 a]}$, 通过 1-甲基-3-乙酰基-4-差弪 基-2-喹啉酮(1)和芳香醛的 Aldol 缩合反应可以得到较 好收率，但反应时间长 $(3 \mathrm{~d})$. 超声波技术的应用使得该 反应得以改进, 反应时间缩短为 $1.5 \mathrm{~h}^{[16 b]}$, 收率良好, 成 为一种合成该类化合物的有效方法.

本实验方法以水代替有机溶剂，在超声波辐射下， 通过哌啶与聚乙二醇(PEG-400)的协同作用, 以较高收

\footnotetext{
* Corresponding author. E-mail: wangdaolin@sina.com

Received December 1, 2016; revised January 11, 2017; published online February 17, 2017

Project supported by the Innovation Team Project of Liaoning Province Education Department (No. 2015001).

辽宁省教育厅创新团队(No. 2015001)资助项目.
} 
<smiles>CC(=O)c1c(O)c2ccccc2n(C)c1=O</smiles>

图式 1 合成路线

Scheme 1 Synthetic route

率制备了 3-肉桂酰基-4-羟基-2-喹啉酮(3). 在这一研究 工作中, 我们考察了反应条件对该反应的影响(表 1), 发 现 $10 \mathrm{~mol} \%$ 的哌啶与 $2.5 \%$ 的 PEG-400 用量比较合适, 反 应可以在 $2 \mathrm{~h}$ 内完成(Entry 4), 产率较高; 与已有方法相 比(Entries 1 3), 具有明显优势; 使用更多的催化剂(20 mol\%的哌啶与 5\%的 PEG-400)时, 产物收率差别不大 (Entry 5); 当使用 $\mathrm{EtOH}$ 作为反应溶剂时, $2 \mathrm{~h}$ 后, $\mathbf{3 a}$ 的收 率为 53\% (Entry 6); 无 PEG-400 参与时 3a 收率显著下 降(Entry 7). 进一步证明 PEG-400 在 Aldol 缩合反应中 的有效性 ${ }^{[17]}$.

在此基础上, 我们选择以哌啶 (10 mol\%) 与 PEG-400 (2.5 mol\%)作为该反应的协同催化剂, 在超声 波辅助下, 于 $80{ }^{\circ} \mathrm{C}$ 合成 3-肉桂酰基-4-羊基-2-喹啉酮, 实验结果列于表 2 .

\section{2 吡喃并[3,2-c]喹啉-4,5-二酮类衍生物的合成}

在黄酮及其相关类似物的合成过程中利用 $\mathrm{I}_{2} / \mathrm{DMSO}$ 体系是一种重要的合成途径 ${ }^{[18]}$. 我们曾经报道
物的合成方法 ${ }^{[17 a]}$. 作为该方法的延续, 本工作旨在探 讨 $\mathrm{I}_{2}$ 对 1-甲基-3-肉桂酰基-4-羊基-2-喹啉酮类化合物的 环化反应性能. 首先以 1-甲基-3-肉桂酰基-4-差基-2-喹 啉酮(3a)为反应底物, 主要考察溶剂、催化剂用量和反 应温度对反应的影响, 结果见表 3. 实验发现在 $100{ }^{\circ} \mathrm{C}$ 下无任何催化剂参与时, 该反应无法进行(Entry 1); 在 $\mathrm{I}_{2}$ 存在下反应可以较为顺利地进行 (Entries $2 \sim 6$ ). 其中, 碘的用量为 $15 \mathrm{~mol} \%$ 时(Entry 4), 目标产物收率可达到 $84 \%$. 增加催化剂的用量 $(20 \mathrm{~mol} \%)$ 或升高反应温度 $\left(110{ }^{\circ} \mathrm{C}\right)$, 产物收率并没有得到改善(Entries 5,6). 其次, 通过与 $N, N$-二甲基甲酰胺(DMF) 和 HOAc 为反应溶剂的 比较(Entries 7,8), 发现二甲基亚砜(DMSO)作为溶剂效 果较佳, 可能源于良好的溶解性能以及弱氧化性作 用 ${ }^{[19]}$. 因此选择以 DMSO 为反应溶剂、碘的用量为 15 $\mathrm{mol} \%$ 作为该反应的优化条件.

在以上优化反应条件下, 对吡喃并 [3,2-c]喹啉-4,5二酮类衍生物(4)的合成进行扩展实验(表 4).

从反应结果中可以看出，1-甲基-3-肉桂酰基-4-羟 基-2-喹啉酮类化合物在 $\mathrm{I}_{2} / \mathrm{DMSO}$ 体系中, 表现出较高 的反应活性, 可以顺利实现环化: 当苯环上含有供电子 取代基(如 $\mathrm{Me}, \mathrm{OMe}, \mathrm{OH}$ )时(Entries 2 8), 表现出良好 的选择性和高的收率 $(82 \sim 88 \%$ ); 含有吸电子取代基(如 $\mathrm{Cl}, \mathrm{F}$ )时(Entries 9, 10), 也得到良好收率 $(78 \%, 75 \%)$. 另 外, 杂环芳香醛、如糠醛和噻吩-2-甲醛(Entries 11，12) 应用于该反应也得到较好收率 $(70 \%, 72 \%)$.

所制备产物结构经 IR、 ${ }^{1} \mathrm{H} \mathrm{NMR} 、{ }^{13} \mathrm{C}$ NMR 和元素 分析数据得以确认. 在 IR 谱图中, 目标化合物吡喃并 [3,2-c]喹啉-4,5-二酮(4)的共同特征为在 $1756 \sim 1771$ $\mathrm{cm}^{-1}$ 范围内表现出喹啉酮羰基的伸缩振动吸收峰, 在 了在 $\mathrm{I}_{2} / \mathrm{DMSO}$ 体系中香豆素并[4,3- $b$ ] 吡喃-4-酮类衍生

表 1 3-肉桂酰基-4-羟基-2-喹啉酮合成条件的优化

Table 1 Optimization of reaction conditions on the synthesis of 1-methyl-3-cinnamoyl-4-hydroxyquinoline-2-one (3a) ${ }^{a}$

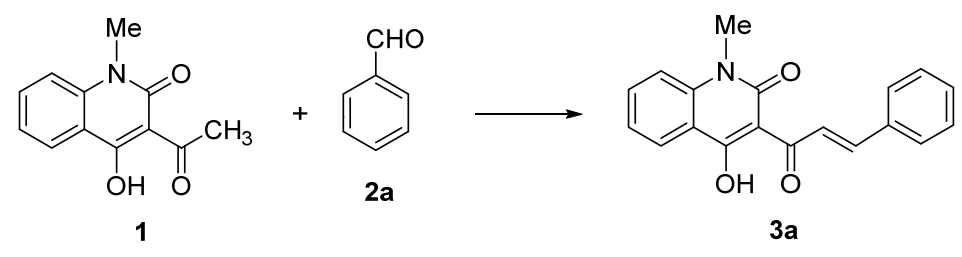

\begin{tabular}{clclcc}
\hline \multicolumn{1}{c}{ Catalyst } & Solvent & \multicolumn{1}{c}{ Conditions } & Time $^{b} / \mathrm{h}$ & Yield $/ \%$ \\
\hline Entry & \multicolumn{1}{c}{ EtOH } & $80{ }^{\circ} \mathrm{C}$ & 72 & $25^{[16 a]}$ \\
2 & P-Proline $(30 \mathrm{~mol} \%)$ & $\mathrm{BuOH}$ & Reflux & 6 & $38^{[16 \mathrm{~b}]}$ \\
3 & $\mathrm{Al}_{2} \mathrm{O}_{3}$ & $\mathrm{BuOH}$ & Ultrasonic irradiation, $60{ }^{\circ} \mathrm{C}$ & 1.5 & $83^{[16 \mathrm{~b}]}$ \\
4 & Piperidine $(10 \mathrm{~mol} \%) /$ PEG-400 $(2.5 \mathrm{~mol} \%)$ & $\mathrm{H}_{2} \mathrm{O}$ & Ultrasonic irradiation, $80{ }^{\circ} \mathrm{C}$ & 2 & 85 \\
5 & Piperidine $(20 \mathrm{~mol} \%) /$ PEG-400 $(5 \mathrm{~mol} \%)$ & $\mathrm{H}_{2} \mathrm{O}$ & Ultrasonic irradiation, $80{ }^{\circ} \mathrm{C}$ & 2 & 86 \\
6 & Piperidine $(10 \mathrm{~mol} \%) /$ PEG-400 $(2.5 \mathrm{~mol} \%)$ & $\mathrm{EtOH}$ & Ultrasonic irradiation, $80{ }^{\circ} \mathrm{C}$ & 2 & 53 \\
7 & Piperidine $(10 \mathrm{~mol} \%)$ & $\mathrm{H}_{2} \mathrm{O}$ & Ultrasonic irradiation, $80{ }^{\circ} \mathrm{C}$ & 2 & 67 \\
\hline
\end{tabular}

${ }^{a}$ Reaction conditions: 1-methyl-3-acetyl-4-hydroxyquinoline-2-one $(1,1 \mathrm{mmol})$, solvent $(10 \mathrm{~mL}) ;{ }^{b}$ Reaction progress was monitored by TLC. ${ }^{c}$ Isolated yield. 
表 2 化合物 3 的反应时间及产率

Table 2 Reaction times and yields of the products $\mathbf{3}^{a}$<smiles>CC(=O)c1c(O)c2ccccc2n(C)c1=O</smiles><smiles>Cn1c(=O)c(C(=O)/C=C/[Al])c(O)c2ccccc21</smiles>

\begin{tabular}{|c|c|c|c|c|}
\hline Entry & $\mathrm{Ar}$ & Product 3 & Time $^{b} / \mathrm{h}$ & Yield $/{ }^{c} \%$ \\
\hline 1 & $\mathrm{Ph}$ & $\mathbf{3 a}$ & 2 & 85 \\
\hline 2 & 4- $\mathrm{MeC}_{6} \mathrm{H}_{4}$ & $3 \mathbf{b}$ & 3 & 83 \\
\hline 3 & 2- $\mathrm{MeOC}_{6} \mathrm{H}_{4}$ & $3 c$ & 3 & 79 \\
\hline 4 & $3-\mathrm{MeOC}_{6} \mathrm{H}_{4}$ & 3d & 3 & 87 \\
\hline 5 & 4- $\mathrm{MeOC}_{6} \mathrm{H}_{4}$ & $3 e$ & 2 & 85 \\
\hline 6 & 2,4-(MeO) $)_{2} \mathrm{C}_{6} \mathrm{H}_{3}$ & $3 f$ & 2 & 80 \\
\hline 7 & $3,4-(\mathrm{MeO})_{2} \mathrm{C}_{6} \mathrm{H}_{3}$ & $3 g$ & 1.5 & 86 \\
\hline 8 & $2,4,5-(\mathrm{MeO}){ }_{3} \mathrm{C}_{6} \mathrm{H}_{2}$ & $3 \mathrm{~h}$ & 1.5 & 82 \\
\hline 9 & $4-\mathrm{ClC}_{6} \mathrm{H}_{4}$ & $3 \mathbf{i}$ & 3 & 80 \\
\hline 10 & $2-\mathrm{FC}_{6} \mathrm{H}_{4}$ & $3 \mathbf{j}$ & 3 & 75 \\
\hline 11 & 2-Furyl & $3 \mathbf{k}$ & 3 & 77 \\
\hline 12 & 2-Thienyl & 31 & 3 & 75 \\
\hline
\end{tabular}

${ }^{a}$ Reaction conditions: 1-methyl-3-acetyl-4-hydroxyquinoline-2-one (1, 10.0 $\mathrm{mmol}), \mathrm{H}_{2} \mathrm{O}(10 \mathrm{~mL})$, aldehyde $(2,12.0 \mathrm{mmol})$, pyridine $(1.0 \mathrm{mmol})$, PEG-400 (100 mg), $80{ }^{\circ} \mathrm{C} ;{ }^{b}$ Reaction progress was monitored by TLC. ${ }^{c}$ Isolated yield.

表 3 吡喃并[3,2-c]喹啉-4,5-二酮(4a)合成条件的优化 ${ }^{a}$

Table 3 Optimization of reaction conditions on the synthesis of pyrano[3,2-c]quinoline-4,5-dione (4a)

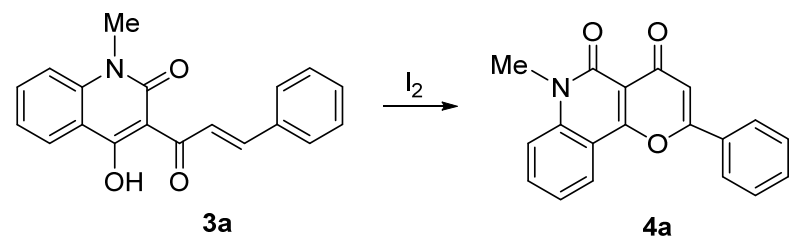

\begin{tabular}{ccccc}
\hline Entry & $\mathrm{I}_{2} / \mathrm{mol} \%$ & Solvent & $\mathrm{Time}^{b} / \mathrm{h}$ & Yield $^{c} \%$ \\
\hline 1 & 0 & DMSO & 24 & $0^{d}$ \\
2 & 5 & DMSO & 24 & 38 \\
3 & 10 & DMSO & 16 & 73 \\
4 & 15 & DMSO & 10 & 84 \\
5 & 20 & DMSO & 9 & 82 \\
6 & 15 & DMSO & 9 & 80 \\
7 & 15 & DMF & 18 & 67 \\
8 & 15 & HOAc & 8 & 54 \\
\hline
\end{tabular}

${ }^{a}$ Reaction conditions: 1-methyl-3-cinnamoyl-4-hydroxyquinoline-2-one (3a, 1 mmol), solvent $(10 \mathrm{~mL}), 100{ }^{\circ} \mathrm{C} ;{ }^{b}$ Reaction progress was monitored by TLC. ${ }^{c}$ Isolated yield. ${ }^{d}$ The starting materials were recovered.

1632 1644 $\mathrm{cm}^{-1}$ 范围内出现的吸收峰为吡喃-4-酮羰基 的伸缩振动吸收峰. 在 ${ }^{1} \mathrm{H}$ NMR 谱图中, 目标产物 4 在 $\delta 6.76 \sim 7.23$ 处的单峰吸收, 为吡喃酮环 3-位质子的特 征吸收峰. 在 ${ }^{13} \mathrm{C} \mathrm{NMR}$ 谱图中, 目标产物 4, 在 $\delta 174 \sim$ 177 处的吸收为喹啉酮羰基碳的特征吸收峰, $\delta 159 \sim$ 162 为吡喃酮羰基碳的特征吸收峰.

依据相关文献 $[11 \mathrm{a}]$ 及实验结果, 推测该反应进行

的可能机理如下(Scheme 2): 首先 1-甲基-3-肉桂酰基-4表 4 化合物 4 的反应时间及产率

Table 4 Reaction times and yields of the products 4

\begin{tabular}{clccc}
\hline Entry & \multicolumn{1}{c}{$\mathrm{Ar}$} & Product $\mathbf{4}$ & Time/h & Yield/\% \\
\hline 1 & $\mathrm{Ph}$ & $\mathbf{4 a}$ & 7 & 84 \\
2 & $4-\mathrm{MeC}_{6} \mathrm{H}_{4}$ & $\mathbf{4 b}$ & 6 & 86 \\
3 & $2-\mathrm{MeOC}_{6} \mathrm{H}_{4}$ & $\mathbf{4 c}$ & 8 & 82 \\
4 & $3-\mathrm{MeOC}_{6} \mathrm{H}_{4}$ & $\mathbf{4 d}$ & 6 & 85 \\
5 & $4-\mathrm{MeOC}_{6} \mathrm{H}_{4}$ & $\mathbf{4 e}$ & 5 & 87 \\
6 & $2,4-(\mathrm{MeO})_{2} \mathrm{C}_{6} \mathrm{H}_{3}$ & $\mathbf{4 f}$ & 7 & 85 \\
7 & $3,4-(\mathrm{MeO})_{2} \mathrm{C}_{6} \mathrm{H}_{3}$ & $\mathbf{4 g}$ & 5 & 88 \\
8 & $2,4,5-(\mathrm{MeO})_{3} \mathrm{C}_{6} \mathrm{H}_{2}$ & $\mathbf{4 h}$ & 6 & 85 \\
9 & $4-\mathrm{ClC}_{6} \mathrm{H}_{4}$ & $\mathbf{4 i}$ & 10 & 78 \\
10 & $2-\mathrm{FC}_{6} \mathrm{H}_{4}$ & $\mathbf{4 j}$ & 12 & 75 \\
11 & $2-F u r y l$ & $\mathbf{4 k}$ & 9 & 70 \\
12 & $2-\mathrm{Thienyl}^{2}$ & $\mathbf{4 l}$ & 8 & 72 \\
\hline
\end{tabular}

着弪基 2-喹啉酮(3)在分子碘的作用下，通过加成反应得 到碘代鎓盐 $\mathbf{A}$ ，接着通过分子内亲核取代得到环化产物 $\mathbf{B}$, 最后经过分子内脱除一分子 $\mathrm{HI}$ 得到最终产物: 吡喃 并 $[3,2-c]$ 喹啉-4,5-二酮(4).<smiles>Cn1c(=O)c(C(=O)/C=C/[Al])c(O)c2ccccc21</smiles><smiles></smiles>

图式 2 反应机理

Scheme 2 Reation mechanism

\section{2 结论}

通过 1-甲基-3-乙酰基-4-差基-2-喹啉酮与芳香醛的 Aldol 缩合及在碘促进下的环化反应，成功地合成了一 系列吡喃并 [3,2-c]喹啉-4,5-二酮类化合物, 为新型吡喃 并喹啉酮类衍生物提供了有效的合成策略. 该方法反应 条件温和、操作简便、收率良好，有较好的实用价值及 应用前景.

\section{3 实验部分}

\section{1 仪器与试剂}

熔点用 WRS-1B 数字熔点仪测定, 熔点未校正; NMR 用 Bruker DPX-400 MHz 核磁共振仪测定，四甲基 硅烷为内标; IR 用 BIO-RAD FTS-40 型傅立叶变换红外 
分光光度计测定, $\mathrm{KBr}$ 压片; MS 采用 ZAB-HS 型质谱仪 测定; 元素分析用 Vario EL 元素分析仪测定; 超声波用 频率为 $40 \mathrm{kHz}$ 和 $250 \mathrm{~W}$ 输出功率的 KQ-250B 医用超声 清洁器. 所用试剂均为分析纯试剂, 1-甲基-3-乙酰基-4着基-2-喹啉酮(1)按照文献[20]方法制备.

\section{2 实验方法}

\subsubsection{1-甲基-3-肉桂酰基-4-羟基-2-喹啉酮(3)的制备}

合成的一般步骤: 于超声波辐射下，将 1-甲基-3-乙 酰基-4-在基-2-喹啉酮(1) (2.17 g, $10.0 \mathrm{mmol}$ )、哌啶(85 $\mathrm{mg}, 1.0 \mathrm{mmol})$ 、芳香醶 $(2)(12.0 \mathrm{mmol}) 、$ 水 $(20 \mathrm{~mL})$ 和 PEG-400 (100 mg), 在 $80{ }^{\circ} \mathrm{C}$ 下反应 $1.5 \sim 3 \mathrm{~h}$, 用硅胶层 析板(TLC)监控反应. 反应结束后, 冷却, 过滤, 无水乙 醇重结晶, 得到产物 3 . 产物 $3 \mathrm{a} \sim 31$ 的物理分析数据与 光谱数据如下:

1-甲基-3-肉桂酰基-4-羟基-2-喹啉酮(3a)：黄色针状 晶体. m.p. $155 \sim 157{ }^{\circ} \mathrm{C}$ (文献值 ${ }^{[6 a]} 148 \sim 150{ }^{\circ} \mathrm{C}$ ); ${ }^{1} \mathrm{H}$ NMR $\left(\mathrm{CDCl}_{3}, 400 \mathrm{MHz}\right) \delta: 3.65\left(\mathrm{~s}, 3 \mathrm{H}, \mathrm{OCH}_{3}\right), 7.25 \sim$ $7.31(\mathrm{~m}, 1 \mathrm{H}), 7.38 \sim 7.40(\mathrm{~m}, 3 \mathrm{H}), 7.66 \sim 7.71(\mathrm{~m}, 3 \mathrm{H})$, 7.95 (d, $J=8.0 \mathrm{~Hz}, 1 \mathrm{H}), 8.24(\mathrm{~d}, J=8.0 \mathrm{~Hz}, 1 \mathrm{H}), 8.68(\mathrm{~d}$, $J=8.0 \mathrm{~Hz}, 1 \mathrm{H}) ;{ }^{13} \mathrm{C}$ NMR $\left(\mathrm{CDCl}_{3}, 100 \mathrm{MHz}\right) \delta: 29.2$, $114.2,116.0,122.1,125.2,126.2,128.8,129.4,130.7$, 134.9, 135.1, 141.6, 145.1, 161.6, 176.8, 176.9, 194.4; IR (KBr) v: $3231(\mathrm{OH}), 1654(\mathrm{C}=\mathrm{O}), 1577(\mathrm{C}=\mathrm{O}) \mathrm{cm}^{-1}$. Anal. calcd for $\mathrm{C}_{19} \mathrm{H}_{15} \mathrm{NO}_{3}: \mathrm{C} 74.74, \mathrm{H} 4.95, \mathrm{~N} 4.59$; found C 74.89, H 4.97, N 4.61.

1-甲基-3-(4-甲基肉桂酰基)-4-差基-2-喹啉酮(3b): 黄色针状晶体. m.p. 170 172 ${ }^{\circ} \mathrm{C}$ (文献值 ${ }^{[16 a]} 166 \sim$ $\left.168{ }^{\circ} \mathrm{C}\right) ;{ }^{1} \mathrm{H} \mathrm{NMR}\left(\mathrm{CDCl}_{3}, 400 \mathrm{MHz}\right) \delta: 2.37\left(\mathrm{~s}, 3 \mathrm{H}, \mathrm{CH}_{3}\right)$, $3.66\left(\mathrm{~s}, 3 \mathrm{H}, \mathrm{NCH}_{3}\right), 7.18 \sim 7.30(\mathrm{~m}, 4 \mathrm{H}), .60(\mathrm{~d}, J=7.6$ $\mathrm{Hz}, 2 \mathrm{H}), 7.67$ (dd, $J=7.6,8.4 \mathrm{~Hz}, 1 \mathrm{H}), 8.14$ (d, $J=15.6$ $\mathrm{Hz}, 1 \mathrm{H}), 8.23(\mathrm{~d}, J=8.0 \mathrm{~Hz}, 1 \mathrm{H}), 8.82(\mathrm{~d}, J=15.6 \mathrm{~Hz}$, $1 \mathrm{H}) ;{ }^{13} \mathrm{C}$ NMR $\left(\mathrm{CDCl}_{3}, 100 \mathrm{MHz}\right) \delta: 19.3,26.1,103.2$, $111.8,113.8,119.7,121.7,123.8,126.7,127.3,130.1$, 132.6, 138.9, 139.3, 143.0, 159.4, 173.9, 191.8; IR (KBr) v: $3236(\mathrm{OH}), 1658(\mathrm{C}=\mathrm{O}), 1594(\mathrm{C}=\mathrm{O}) \mathrm{cm}^{-1}$. Anal. calcd for $\mathrm{C}_{20} \mathrm{H}_{17} \mathrm{NO}_{3}$ : C 75.22, $\mathrm{H}$ 5.37, N 4.39; found $\mathrm{C}$ 75.37, H 5.36, N 4.41.

1-甲基-3-(2-甲氧基肉桂酰基)-4-羟基-2-喹啉酮(3c): 黄色针状晶体. m.p. 162 163 ${ }^{\circ} \mathrm{C} ;{ }^{1} \mathrm{H}$ NMR $\left(\mathrm{CDCl}_{3}, 400\right.$ $\mathrm{MHz}) \delta: 3.65$ (s, $\left.3 \mathrm{H}, \mathrm{NCH}_{3}\right), 3.90\left(\mathrm{~s}, 3 \mathrm{H}, \mathrm{OCH}_{3}\right), 6.89 \sim$ $6.98(\mathrm{~m}, 2 \mathrm{H}), 7.22 \sim 7.27(\mathrm{~m}, 2 \mathrm{H}), 7.36(\mathrm{t}, J=7.6 \mathrm{~Hz}, 1 \mathrm{H})$, $7.66(\mathrm{dd}, J=7.8,8.0 \mathrm{~Hz}, 1 \mathrm{H}), 7.80(\mathrm{~d}, J=8.0 \mathrm{~Hz}, 1 \mathrm{H})$, $8.24(\mathrm{~d}, J=8.0 \mathrm{~Hz}, 1 \mathrm{H}), 8.57$ (d, $J=15.6, \mathrm{~Hz}, 1 \mathrm{H}), 8.86$ $(\mathrm{d}, J=15.6 \mathrm{~Hz}, 1 \mathrm{H}) ;{ }^{13} \mathrm{C} \mathrm{NMR}\left(\mathrm{CDCl}_{3}, 100 \mathrm{MHz}\right) \delta: 29.2$, $55.5,105.6,111.4,114.3,116.2,120.7,122.0,124.1$,
$124.9,126.3,128.7,132.1,134.8,140.1 .141 .6,158.7$, 161.8, 176.4, 194.5; IR (KBr) v: $3230(\mathrm{OH}), 1652(\mathrm{C}=\mathrm{O})$, $1589(\mathrm{C}=\mathrm{O}) \mathrm{cm}^{-1}$. Anal. calcd for $\mathrm{C}_{20} \mathrm{H}_{17} \mathrm{NO}_{4}: \mathrm{C} 71.63, \mathrm{H}$ 5.11, N 4.18; found C 71.78, H 5.10, N 4.19.

1-甲基-3-(3-甲氧基肉桂酰基)-4-羟基-2-喹啉酮 (3d): 黄色针状晶体. m.p. $139 \sim 141{ }^{\circ} \mathrm{C} ;{ }^{1} \mathrm{H}$ NMR $\left(\mathrm{CDCl}_{3}, 400 \mathrm{MHz}\right) \delta: 3.66\left(\mathrm{~s}, 3 \mathrm{H}, \mathrm{NCH}_{3}\right), 3.85(\mathrm{~s}, 3 \mathrm{H}$, $\left.\mathrm{OCH}_{3}\right), 6.94(\mathrm{~d}, J=7.6 \mathrm{~Hz}, 1 \mathrm{H}), 7.20 \sim 7.31(\mathrm{~m}, 5 \mathrm{H}), 7.68$ (t, $J=7.6 \mathrm{~Hz}, 1 \mathrm{H}), 7.92$ (d, $J=15.6 \mathrm{~Hz}, 1 \mathrm{H}), 8.24$ (d, $J=$ $7.6 \mathrm{~Hz}, 1 \mathrm{H}), 8.65(\mathrm{~d}, J=15.6 \mathrm{~Hz}, 1 \mathrm{H}) ;{ }^{13} \mathrm{C} \mathrm{NMR}\left(\mathrm{CDCl}_{3}\right.$, $100 \mathrm{MHz}) \delta$ : 29.2, 55.4, 105.6, 113.8, 114.3, 116.1, 116.7, $121.7,122.1,125.5,126.3,129.8,134.9,136.5,141.7$, 145.0, 159.8, 161.8, 176.2, 194.4; IR (KBr) v: $3234(\mathrm{OH})$, $1659(\mathrm{C}=\mathrm{O}), 1582(\mathrm{C}=\mathrm{O}) \mathrm{cm}^{-1}$. Anal. calcd for $\mathrm{C}_{20} \mathrm{H}_{17^{-}}$ $\mathrm{NO}_{4}$ : C 71.63, H 5.11, N 4.18; found C 71.75, H 5.12, N 4.20.

1-甲基-3-(4-甲氧基肉桂酰基)-4-美基-2-喹啉酮(3e): 黄色针状晶体. m.p. $171 \sim 173{ }^{\circ} \mathrm{C}$ (文献值 ${ }^{[16 a]} 170 \sim$ $\left.172{ }^{\circ} \mathrm{C}\right) ;{ }^{1} \mathrm{H}$ NMR $\left(\mathrm{CDCl}_{3}, 400 \mathrm{MHz}\right) \delta: 3.71(\mathrm{~s}, 3 \mathrm{H}$, $\left.\mathrm{NCH}_{3}\right), 3.92\left(\mathrm{~s}, 3 \mathrm{H}, \mathrm{OCH}_{3}\right), 7.05(\mathrm{~d}, J=8.4 \mathrm{~Hz}, 1 \mathrm{H}), 7.14$ (t, $J=7.6,8.0 \mathrm{~Hz}, 1 \mathrm{H}), 7.32 \sim 7.41(\mathrm{~m}, 4 \mathrm{H}), 7.50(\mathrm{~d}, J=$ $7.6,8.0 \mathrm{~Hz}, 1 \mathrm{H}), 7.72(\mathrm{~d}, J=7.6,8.0 \mathrm{~Hz}, 1 \mathrm{H}), 7.76(\mathrm{~d}, J=$ $15.6 \mathrm{~Hz}, 1 \mathrm{H}), 8.24(\mathrm{~d}, J=15.6 \mathrm{~Hz}, 1 \mathrm{H}) ;{ }^{13} \mathrm{C} \mathrm{NMR}\left(\mathrm{CDCl}_{3}\right.$, $100 \mathrm{MHz}) \delta: 27.4,53.6,112.3,112.5,114.5,120.2,120.8$, $124.4,126.2,127.5,129.1,132.9,139.7,143.5,160.0$, 160.1, 174.5, 192.2; IR (KBr) v: $3235(\mathrm{OH}), 1659(\mathrm{C}=\mathrm{O})$, $1586(\mathrm{C}=\mathrm{O}) \mathrm{cm}^{-1}$. Anal. calcd for $\mathrm{C}_{20} \mathrm{H}_{17} \mathrm{NO}_{4}: \mathrm{C} 71.63, \mathrm{H}$ 5.11, N 4.18; Found C 71.74, H 5.13, N 4.20.

1-甲基-3-(2,4-二甲氧基肉桂酰基)-4-羟基-2-喹啉酮 (3f): 黄色针状晶体. m.p. 191 193 ${ }^{\circ} \mathrm{C}$ (文献值 ${ }^{[16 a]} 187 \sim$ $\left.189{ }^{\circ} \mathrm{C}\right) ;{ }^{1} \mathrm{H}$ NMR $\left(\mathrm{CDCl}_{3}, 400 \mathrm{MHz}\right) \delta: 3.66$ (s, 3H, $\left.\mathrm{NCH}_{3}\right), 3.84\left(\mathrm{~s}, 3 \mathrm{H}, \mathrm{OCH}_{3}\right), 3.89\left(\mathrm{~s}, 3 \mathrm{H}, \mathrm{OCH}_{3}\right), 6.43$ (s, $1 \mathrm{H}), 6.50(\mathrm{~d}, J=8.4 \mathrm{~Hz}, 1 \mathrm{H}), 7.22 \sim 7.29(\mathrm{~m}, 2 \mathrm{H}), 7.65(\mathrm{t}$, $J=7.6 \mathrm{~Hz}, 1 \mathrm{H}), 7.76(\mathrm{~d}, J=8.4 \mathrm{~Hz}, 1 \mathrm{H}), 8.23(\mathrm{~d}, J=8.0$ $\mathrm{Hz}, 1 \mathrm{H}), 8.37$ (d, $J=15.6 \mathrm{~Hz}, 1 \mathrm{H}), 8.61$ (d, $J=15.6 \mathrm{~Hz}$, $1 \mathrm{H}) ;{ }^{13} \mathrm{C} \mathrm{NMR}\left(\mathrm{CDCl}_{3}, 100 \mathrm{MHz}\right) \delta: 27.3,53.7,96.2,96.3$, $103.7,112.3,114.6,115.6,120.1,120.2,124.4,128.6$, 132.7, 138.7, 139.2, 158.7, 160.1, 161.7, 174.7, 192.3; IR (KBr) v: $3235(\mathrm{OH}), 1656(\mathrm{C}=\mathrm{O}), 1594(\mathrm{C}=\mathrm{O}) \mathrm{cm}^{-1}$. Anal. calcd for $\mathrm{C}_{21} \mathrm{H}_{19} \mathrm{NO}_{5}$ : C 69.03, H 5.24, N 3.83; found C 69.16, H 5.25, N 3.85 .

1-甲基-3-(3,4-二甲氧基肉桂酰基)-4-羟基-2-喹啉酮 (3g): 黄色针状晶体. m.p. $194 \sim 196{ }^{\circ} \mathrm{C} ;{ }^{1} \mathrm{H}$ NMR $\left(\mathrm{CDCl}_{3}, 400 \mathrm{MHz}\right) \delta: 3.66\left(\mathrm{~s}, 3 \mathrm{H}, \mathrm{NCH}_{3}\right), 3.91(\mathrm{~s}, 3 \mathrm{H}$, $\left.\mathrm{OCH}_{3}\right), 3.95\left(\mathrm{~s}, 3 \mathrm{H}, \mathrm{OCH}_{3}\right), 6.86(\mathrm{~d}, J=7.6 \mathrm{~Hz}, 1 \mathrm{H})$, 
$7.22 \sim 7.30(\mathrm{~m}, 4 \mathrm{H}), 7.67(\mathrm{dd}, J=7.6,8.0 \mathrm{~Hz}, 1 \mathrm{H}), 7.94(\mathrm{~d}$, $J=15.6 \mathrm{~Hz}, 1 \mathrm{H}), 8.23(\mathrm{~d}, J=8.4 \mathrm{~Hz}, 1 \mathrm{H}), 8.56(\mathrm{~d}, J=15.6$ $\mathrm{Hz}, 1 \mathrm{H}) ;{ }^{13} \mathrm{C} \mathrm{NMR}\left(\mathrm{CDCl}_{3}, 100 \mathrm{MHz}\right) \delta: 29.2,55.9,105.4$, $110.5,110.9,114.2,116.3,122.1,122.7,124.0,126.2$, $138.3,134.8,141.6,145.7,149.1,151.6,161.9,176.4$, 193.9; IR (KBr) v: $3230(\mathrm{OH}), 1652(\mathrm{C}=\mathrm{O}), 1589(\mathrm{C}=\mathrm{O})$ $\mathrm{cm}^{-1}$. Anal. calcd for $\mathrm{C}_{21} \mathrm{H}_{19} \mathrm{NO}_{5}: \mathrm{C} 69.03, \mathrm{H} 5.24, \mathrm{~N} 3.83$; found C 69.14, H 5.23, N 3.85 .

1-甲基-3-(2,4,5-三甲氧基肉桂酰基)-4-羟基-2-喹啉 酮(3h): 黄色针状晶体. m.p. $223 \sim 224{ }^{\circ} \mathrm{C} ;{ }^{1} \mathrm{H}$ NMR $\left(\mathrm{CDCl}_{3}, 400 \mathrm{MHz}\right) \delta: 3.65$ (s, $\left.3 \mathrm{H}, \mathrm{NCH}_{3}\right), 3.90(\mathrm{~s}, 3 \mathrm{H}$, $\left.\mathrm{OCH}_{3}\right), 3.91\left(\mathrm{~s}, 3 \mathrm{H}, \mathrm{OCH}_{3}\right), 3.93\left(\mathrm{~s}, 3 \mathrm{H}, \mathrm{OCH}_{3}\right), 6.48$ (s, $1 \mathrm{H}), 7.22 \sim 7.29(\mathrm{~m}, 3 \mathrm{H}), 7.66(\mathrm{dd}, J=7.2,7.6 \mathrm{~Hz}, 1 \mathrm{H})$, $8.23(\mathrm{~d}, J=7.6 \mathrm{~Hz}, \mathrm{H}), 8.42(\mathrm{~d}, J=15.6 \mathrm{~Hz}, 1 \mathrm{H}), 8.56$ (d, $J=15.6 \mathrm{~Hz}, 1 \mathrm{H}) ;{ }^{13} \mathrm{C} \mathrm{NMR}\left(\mathrm{CDCl}_{3}, 100 \mathrm{MHz}\right) \delta: 26.9$, 53.6, 54.1, 54.2, 94.1, 103.1, 108.3, 111.2, 113.6, 114.3, $119.5,119.6,123.8,132.3,138.6,139.3,140.9,150.7$, 152.6, 159.6, 174.2, 191.6; IR (KBr) v: $3227(\mathrm{OH}), 1646$ $(\mathrm{C}=\mathrm{O}), 1589(\mathrm{C}=\mathrm{O}) \mathrm{cm}^{-1}$. Anal. calcd for $\mathrm{C}_{22} \mathrm{H}_{21} \mathrm{NO}_{6}: \mathrm{C}$ 66.83, H 5.35, N 3.54; found C 66.96, H 5.36, N 3.53.

1-甲基-3-(4-氯肉桂酰基)-4-差基-2-喹啉酮(3i)：黄 色针状晶体. m.p. $186 \sim 188{ }^{\circ} \mathrm{C}$ (文献值 ${ }^{[16 b]} 182 \sim$ $\left.184{ }^{\circ} \mathrm{C}\right) .{ }^{1} \mathrm{H}$ NMR $\left(\mathrm{CDCl}_{3}, 400 \mathrm{MHz}\right) \delta: 3.64(\mathrm{~s}, 3 \mathrm{H}$, $\left.\mathrm{NCH}_{3}\right), 7.22 \sim 7.37(\mathrm{~m}, 4 \mathrm{H}), 7.61(\mathrm{~d}, J=8.4 \mathrm{~Hz}, 2 \mathrm{H}), 7.67$ (dd, $J=7.6,8.0 \mathrm{~Hz}, 1 \mathrm{H}), 7.86(\mathrm{~d}, J=15.6 \mathrm{~Hz}, 1 \mathrm{H}), 8.22$ $(\mathrm{d}, J=7.6 \mathrm{~Hz}, 1 \mathrm{H}), 8.63(\mathrm{~d}, J=15.6 \mathrm{~Hz}, 1 \mathrm{H}) ;{ }^{13} \mathrm{C} \mathrm{NMR}$ $\left(\mathrm{CDCl}_{3}, 100 \mathrm{MHz}\right) \delta: 29.2,105.6,114.3,115.9,122.1$, $125.7,126.2,129.7,130.8,133.7,134.9,136.5,141.6$, 143.3, 161.7, 176.7, 194.1; IR (KBr) v: $3234(\mathrm{OH}), 1647$ $(\mathrm{C}=\mathrm{O}), 1583(\mathrm{C}=\mathrm{O}) \mathrm{cm}^{-1}$. Anal. calcd for $\mathrm{C}_{19} \mathrm{H}_{14} \mathrm{ClNO}_{3}$ : C 67.16, H 4.15, N 4.12; found C 67.23, H 4.16, N 4.10.

1-甲基-3-(2-氟肉桂酰基)-4-差基-2-喹啉酮 (3j): 黄 色针状晶体. m.p. $223 \sim 224{ }^{\circ} \mathrm{C} ;{ }^{1} \mathrm{H}$ NMR $\left(\mathrm{CDCl}_{3}, 400\right.$ MHz) $\delta: 3.71\left(3 \mathrm{H}, \mathrm{NCH}_{3}, \mathrm{~s}\right), 7.13 \sim 7.17(\mathrm{~m}, 2 \mathrm{H}), 7.21 \sim$ $7.42(\mathrm{~m}, 3 \mathrm{H}), 7.74$ (t, $J=7.6 \mathrm{~Hz}, 1 \mathrm{H}), 7.85$ (t, $J=6.8 \mathrm{~Hz}$, $1 \mathrm{H}), 8.17$ (d, $J=8.0 \mathrm{~Hz}, 1 \mathrm{H}), 8.29$ (d, $J=7.6 \mathrm{~Hz}, 1 \mathrm{H}), 8.76$ $(\mathrm{d}, J=15.6 \mathrm{~Hz}, 1 \mathrm{H}) ;{ }^{13} \mathrm{C} \mathrm{NMR}\left(\mathrm{CDCl}_{3}, 100 \mathrm{MHz}\right) \delta: 27.4$, $112.4,114.0,114.4,120.3,121.4,122.5,124.4,125.4$, $127.2,130.2,133.2,134.8,139.9,158.6,159.9,161.1$, 174.3, 192.5; IR (KBr) v: $3233(\mathrm{OH}), 1668(\mathrm{C}=\mathrm{O}), 1580$ $(\mathrm{C}=\mathrm{O}) \mathrm{cm}^{-1}$. Anal. calcd for $\mathrm{C}_{19} \mathrm{H}_{14} \mathrm{FNO}_{3}: \mathrm{C} 70.58, \mathrm{H}$ 4.36, N 4.33; found C 70.75, H 4.38, N 4.34.

1-甲基-3-(3-(2-呋喃基)烯丙酰基)-4-羟基-2-喹啉酮 (3k): 黄色针状晶体. m.p. $168 \sim 170{ }^{\circ} \mathrm{C}$ (文献值 ${ }^{[16 c]}$ $\left.160 \sim 162{ }^{\circ} \mathrm{C}\right) ;{ }^{1} \mathrm{H} \mathrm{NMR}\left(\mathrm{CDCl}_{3}, 400 \mathrm{MHz}\right) \delta: 3.65$ (s, $3 \mathrm{H}$,
$\left.\mathrm{NCH}_{3}\right), 6.50(\mathrm{~s}, 1 \mathrm{H}), 6.75(\mathrm{~d}, J=2.8 \mathrm{~Hz}, 1 \mathrm{H}), 7.22 \sim 7.29$ (m, $2 \mathrm{H}), 7.54 \sim 7.55(\mathrm{~m}, 1 \mathrm{H}), 7.65 \sim 7.73(\mathrm{~m}, 2 \mathrm{H}), 8.22(\mathrm{~d}$, $J=8.0 \mathrm{~Hz}, 1 \mathrm{H}), 8.51(\mathrm{~d}, J=15.6 \mathrm{~Hz}, 1 \mathrm{H}) ;{ }^{13} \mathrm{C} \mathrm{NMR}$ $\left(\mathrm{CDCl}_{3}, 100 \mathrm{MHz}\right) \delta: 29.2,112.3,112.7,114.2,116.1$, $116.6,122.5,122.7,126.3,130.9,134.8,141.3,145.5$, 152.1, 161.7, 176.1, 193.7; IR (KBr) v: $3231(\mathrm{OH}), 1656$ $(\mathrm{C}=\mathrm{O}), 1582(\mathrm{C}=\mathrm{O}) \mathrm{cm}^{-1}$. Anal. calcd for $\mathrm{C}_{17} \mathrm{H}_{13} \mathrm{NO}_{4}$ : $\mathrm{C}$ 69.15, H 4.44, N 4.74; found C 69.26, H 4.45, N 4.73.

1-甲基-3-(3-(2-噻吩基)烯丙酰基)-4-差基-2-喹啉酮 (3I): 黄色针状晶体. m.p. $211 \sim 212{ }^{\circ} \mathrm{C} ;{ }^{1} \mathrm{H} \mathrm{NMR}\left(\mathrm{CDCl}_{3}\right.$, $400 \mathrm{MHz}) \delta: 3.66\left(\mathrm{~s}, 3 \mathrm{H}, \mathrm{NCH}_{3}\right), 7.08(\mathrm{~d}, J=7.6,8.4 \mathrm{~Hz}$, $1 \mathrm{H}), 7.27 \sim 7.40(\mathrm{~m}, 2 \mathrm{H}), 7.44 \sim 7.45(\mathrm{~m}, 2 \mathrm{H}), 7.68(\mathrm{dd}$, $J=7.6,8.0 \mathrm{~Hz}, 1 \mathrm{H}), 8.10$ (d, $J=15.6 \mathrm{~Hz}, 1 \mathrm{H}), 8.23(\mathrm{~d}, J=$ $8.0 \mathrm{~Hz}, 1 \mathrm{H}), 8.51(\mathrm{~d}, J=15.6 \mathrm{~Hz}, 1 \mathrm{H}) ;{ }^{13} \mathrm{C} \mathrm{NMR}\left(\mathrm{CDCl}_{3}\right.$, $100 \mathrm{MHz}) \delta: 29.2,105.4,114.2,116.2,122.1,123.8,126.2$, $128.3,129.1,132.3,134.9,137.7,141.0,141.7,161.8$, 176.2, 197.6; IR (KBr) v: $3226(\mathrm{OH}), 1650(\mathrm{C}=\mathrm{O}), 1584$ $(\mathrm{C}=\mathrm{O}) \mathrm{cm}^{-1}$. Anal. calcd for $\mathrm{C}_{17} \mathrm{H}_{13} \mathrm{NO}_{3} \mathrm{~S}: \mathrm{C} 65.58, \mathrm{H}$ 4.21, N 4.50; found C 65.74, H 4.22, N 4.52.

\subsection{2 吡喃并 $[3,2-c]$ 喹啉-4,5-二酮(4)的合成}

合成的一般步骤: 将磾 $(38.00 \mathrm{mg}, 0.15 \mathrm{mmol})$ 加入 到 1-甲基-3-肉桂酰基-4-差基-2-喹啉酩(3) (1.00 mmol) 的 DMSO $\left(10 \mathrm{~mL}\right.$ ) 溶剂中, $100{ }^{\circ} \mathrm{C}$ 下反应 $5 \sim 12 \mathrm{~h}$ (TLC 跟踪检测). 反应完毕后, 将反应混合物转入水中 (50 $\mathrm{mL}$ ), 过滤, 水洗涤, 干燥, 用冰乙酸重结晶, 得到目标 化合物 4. 产物 $4 \mathrm{a} \sim 4 \mathrm{l}$ 的物理分析数据与光谱数据如 下:

1-甲基-2-苯基-6-甲基吡喃并 [3,2-c]喹啉-4,5-二酮 (4a): 黄色针状晶体. m.p. $239 \sim 241{ }^{\circ} \mathrm{C} ;{ }^{1} \mathrm{H}$ NMR $\left(\mathrm{CDCl}_{3}, 400 \mathrm{MHz}\right) \delta: 3.71\left(\mathrm{~s}, 3 \mathrm{H}, \mathrm{NCH}_{3}\right), 6.88(\mathrm{~s}, 1 \mathrm{H})$, $7.34 \sim 7.38(\mathrm{~m}, 2 \mathrm{H}), 7.41 \sim 7.43(\mathrm{~m}, 3 \mathrm{H}), 7.76(\mathrm{t}, J=6.6$ $\mathrm{Hz}, 1 \mathrm{H}), 7.86 \sim 7.87(\mathrm{~m}, 2 \mathrm{H}), 8.25(\mathrm{~d}, J=6.8 \mathrm{~Hz}, 1 \mathrm{H}) ;{ }^{13} \mathrm{C}$ NMR $\left(\mathrm{CDCl}_{3}, 100 \mathrm{MHz}\right) \delta$ : 27.6, 109.1, 110.9, 111.0, $111.3,112.9,120.0,122.3,124.1,127.5,128.8,130.6$, 132.4, 138.9, 159.1, 160.1, 174.2; IR (KBr) $v: 1756(\mathrm{C}=$ O), $1639(\mathrm{C}=\mathrm{O}) \mathrm{cm}^{-1}$. Anal. calcd for $\mathrm{C}_{19} \mathrm{H}_{13} \mathrm{NO}_{3}: \mathrm{C}$ 75.24, H 4.32, N 4.62; found C 75.35, H 4.34, N 4.61.

1-甲基-2-(4-甲基苯基)-6-甲基吡喃并 [3,2-c]喹啉4,5-二䣳(4b): 黄色针状晶体. m.p. $263 \sim 265{ }^{\circ} \mathrm{C} ;{ }^{1} \mathrm{H}$ $\mathrm{NMR}\left(\mathrm{CDCl}_{3}, 400 \mathrm{MHz}\right) \delta: 2.44\left(\mathrm{~s}, 3 \mathrm{H}, \mathrm{CH}_{3}\right), 3.71(\mathrm{~s}, 1 \mathrm{H}$, $\left.\mathrm{NCH}_{3}\right), 6.84(\mathrm{~s}, 1 \mathrm{H}), 7.32 \sim 7.40(\mathrm{~m}, 4 \mathrm{H}), 7.71 \sim 7.76(\mathrm{~m}$, $3 \mathrm{H}), 8.24(\mathrm{~d}, J=8.0 \mathrm{~Hz}, 1 \mathrm{H}) ;{ }^{13} \mathrm{C} \mathrm{NMR}\left(\mathrm{CDCl}_{3}, 100 \mathrm{MHz}\right)$ $\delta$ : 19.2, 27.0, 107.6, 109.2, 109.6, 110.8, 112.4, 120.2, $121.8,123.5,125.4,127.7,131.8,138.4,140.3,158.9$, 159.5, 173.8; IR (KBr) v: $1759(\mathrm{C}=\mathrm{O}), 1632(\mathrm{C}=\mathrm{O})$ 
$\mathrm{cm}^{-1}$. Anal. calcd for $\mathrm{C}_{20} \mathrm{H}_{15} \mathrm{NO}_{3}$ : C 75.70, H 4.76, N 4.41; found C 75.85, H 4.77, N 4.40.

1-甲基-2-(2-甲氧基苯基)-6-甲基吡喃并 [3,2-c]喹啉4,5-二酩 (4c): 黄色针状晶体. m.p. $253 \sim 255{ }^{\circ} \mathrm{C} ;{ }^{1} \mathrm{H}$ NMR $\left(\mathrm{CDCl}_{3}, 400 \mathrm{MHz}\right) \delta: 3.71\left(\mathrm{~s}, 3 \mathrm{H}, \mathrm{NCH}_{3}\right), 3.92(\mathrm{~s}$, $\left.1 \mathrm{H}, \mathrm{OCH}_{3}\right), 7.05(\mathrm{~d}, J=8.4 \mathrm{~Hz}, 1 \mathrm{H}), 7.13(\mathrm{t}, J=7.6 \mathrm{~Hz}$, $1 \mathrm{H}), 7.23(\mathrm{~s}, 1 \mathrm{H}), 7.32 \sim 7.41(\mathrm{~m}, 2 \mathrm{H}), 7.50(\mathrm{t}, J=7.6 \mathrm{~Hz}$, 1H), $7.72(\mathrm{dd}, J=7.6,7.8 \mathrm{~Hz}, 1 \mathrm{H}), 7.88(\mathrm{~d}, J=7.6 \mathrm{~Hz}$, $1 \mathrm{H}), 8.21(\mathrm{~d}, J=8.0 \mathrm{~Hz}, 1 \mathrm{H}) ;{ }^{13} \mathrm{C} \mathrm{NMR}\left(\mathrm{CDCl}_{3}, 100 \mathrm{MHz}\right)$ $\delta:$ 27.6, 53.9, 109.4, 110.1, 111.5, 112.9, 115.4, 117.5, $119.1,120.7,122.4,126.9,131.1,132.3,138.9,156.3$, 156.8, 156.9, 160.3, 174.9; IR (KBr) $v: 1762(\mathrm{C}=\mathrm{O}), 1636$ $(\mathrm{C}=\mathrm{O}) \mathrm{cm}^{-1}$. Anal. calcd for $\mathrm{C}_{20} \mathrm{H}_{15} \mathrm{NO}_{3}: \mathrm{C} 72.06, \mathrm{H} 4.54$, N 4.20; found C 72.17, H 4.56, N 4.21.

1-甲基-2-(3-甲氧基苯基)-6-甲基吡喃并 [3,2-c]喹啉4,5-二酮 (4d): 黄色针状晶体. m.p. $240 \sim 242{ }^{\circ} \mathrm{C} ;{ }^{1} \mathrm{H}$ NMR $\left(\mathrm{CDCl}_{3}, 400 \mathrm{MHz}\right) \delta: 3.72$ (s, 3H, $\left.\mathrm{NCH}_{3}\right), 3.89$ (s, $\left.3 \mathrm{H}, \mathrm{OCH}_{3}\right), 6.89(\mathrm{~s}, 1 \mathrm{H}), 7.09(\mathrm{~s}, 1 \mathrm{H}), 7.35 \sim 7.47(\mathrm{~m}, 4 \mathrm{H})$, $7.45(\mathrm{~d}, J=8.0 \mathrm{~Hz}, 2 \mathrm{H}), 8.25(\mathrm{~d}, J=8.0 \mathrm{~Hz}, 1 \mathrm{H}) ;{ }^{13} \mathrm{C}$ NMR $\left(\mathrm{CDCl}_{3}, 100 \mathrm{MHz}\right) \delta$ : 27.6, 53.7, 109.7, 109.9, $111.1,111.4,112.9,115.3,116.5,120.7,122.3,128.6$, 130.2, 132.4, 139.0, 156.7, 158.3, 158.9, 160.1, 174.2; IR $(\mathrm{KBr}) v$ : $1760(\mathrm{C}=\mathrm{O}), 1633(\mathrm{C}=\mathrm{O}) \mathrm{cm}^{-1}$. Anal. calcd for $\mathrm{C}_{20} \mathrm{H}_{15} \mathrm{NO}_{3}$ : C 72.06, H 4.54, N 4.20; found C 72.15, H $4.56, \mathrm{~N} 4.21$.

1-甲基-2-(4-甲氧基苯基)-6-甲基吡喃并 [3,2-c]喹啉4,5-二酮 (4e): 黄色针状晶体. m.p. $263 \sim 265{ }^{\circ} \mathrm{C} ;{ }^{1} \mathrm{H}$ NMR $\left(\mathrm{CDCl}_{3}, 400 \mathrm{MHz}\right) \delta: 3.75\left(\mathrm{~s}, 3 \mathrm{H}, \mathrm{CH}_{3}\right), 3.91(\mathrm{~s}, 3 \mathrm{H}$, $\left.\mathrm{OCH}_{3}\right), 6.80(\mathrm{~s}, 1 \mathrm{H}), 7.05(\mathrm{~d}, J=8.8 \mathrm{~Hz}, 2 \mathrm{H}), 7.30 \sim 7.42$ (m, 2H), $7.75(\mathrm{t}, J=7.6 \mathrm{~Hz}, 1 \mathrm{H}), 7.83(\mathrm{~d}, J=8.8 \mathrm{~Hz}, 2 \mathrm{H})$, $8.26(\mathrm{~d}, J=8.0 \mathrm{~Hz}, 1 \mathrm{H}) ;{ }^{13} \mathrm{C} \mathrm{NMR}\left(\mathrm{CDCl}_{3}, 100 \mathrm{MHz}\right) \delta$ : 29.3, 55.5, 111.0, 111.1, 111.5, 113.2, 114.6, 122.4, 122.8, $124.1,127.5,134.0,140.7,158.5,160.9,161.7,162.4$, 175.9; IR (KBr) v: $1765(\mathrm{C}=\mathrm{O}), 1639(\mathrm{C}=\mathrm{O}) \mathrm{cm}^{-1}$. Anal. calcd for $\mathrm{C}_{20} \mathrm{H}_{15} \mathrm{NO}_{3}$ : C 72.06, $\mathrm{H} 4.54, \mathrm{~N} 4.20$; found $\mathrm{C}$ $72.19, \mathrm{H} 4.56, \mathrm{~N} 4.19$.

1-甲基-2-(2,4-二甲氧基苯基)-6-甲基吡喃并 [3,2-c] 喹啉-4,5-二酮(4f): 黄色针状晶体. m.p. $268 \sim 269{ }^{\circ} \mathrm{C} ;{ }^{1} \mathrm{H}$ NMR $\left(\mathrm{CDCl}_{3}, 400 \mathrm{MHz}\right) \delta: 3.70$ (s, $\left.3 \mathrm{H}, \mathrm{NCH}_{3}\right), 3.88$ (s, $\left.3 \mathrm{H}, \mathrm{OCH}_{3}\right), 3.89\left(\mathrm{~s}, 3 \mathrm{H}, \mathrm{OCH}_{3}\right), 6.52(\mathrm{~s}, 1 \mathrm{H}), 6.64(\mathrm{~d}, J=$ $8.4 \mathrm{~Hz}, 1 \mathrm{H}), 7.16(\mathrm{~s}, 1 \mathrm{H}), 7.30 \sim 7.38(\mathrm{~m}, 2 \mathrm{H}), 7.70(\mathrm{dd}$, $J=7.4,8.0 \mathrm{~Hz}, 1 \mathrm{H}), 7.83(\mathrm{~d}, J=8.4 \mathrm{~Hz}, 1 \mathrm{H}), 8.18(\mathrm{~d}, J=$ $8.0 \mathrm{~Hz}, 1 \mathrm{H}) ;{ }^{13} \mathrm{C}$ NMR $\left(\mathrm{CDCl}_{3}, 100 \mathrm{MHz}\right) \delta: 27.4,53.8$, $97.1,103.6,109.6,110.5,111.7,112.7,114.2,120.5$, $122.3,127.9,131.9,138.8,156.5,156.6,157.8,159.9$,
161.5, 174.8; IR (KBr) v: $1768(\mathrm{C}=\mathrm{O}), 1635(\mathrm{C}=\mathrm{O})$ $\mathrm{cm}^{-1}$. Anal. calcd for $\mathrm{C}_{21} \mathrm{H}_{17} \mathrm{NO}_{5}: \mathrm{C} 69.41, \mathrm{H} 4.72, \mathrm{~N} 3.85$; found C $69.56, \mathrm{H} 4.73, \mathrm{~N} 3.84$.

1-甲基-2-(3,4-二甲氧基苯基)-6-甲基吡喃并 [3,2-c ] 喹啉-4,5-二酮 $(4 \mathrm{~g})$ : 黄色针状晶体. m.p. $>300{ }^{\circ} \mathrm{C} ;{ }^{1} \mathrm{H}$ NMR $\left(\mathrm{CDCl}_{3}, 400 \mathrm{MHz}\right) \delta: 3.72\left(\mathrm{~s}, 3 \mathrm{H}, \mathrm{NCH}_{3}\right), 3.94(\mathrm{~s}$, $\left.3 \mathrm{H}, \mathrm{OCH}_{3}\right), 3.96\left(\mathrm{~s}, 3 \mathrm{H}, \mathrm{OCH}_{3}\right), 6.84(\mathrm{~s}, 1 \mathrm{H}), 7.01(\mathrm{~d}, J=$ $8.4 \mathrm{~Hz}, 1 \mathrm{H}), 7.27(\mathrm{~s}, 1 \mathrm{H}), 7.37 \sim 7.42(\mathrm{~m}, 2 \mathrm{H}), 7.55(\mathrm{~d}, J=$ $7.6 \mathrm{~Hz}, 1 \mathrm{H}), 7.75(\mathrm{~d}, J=7.6 \mathrm{~Hz}, 1 \mathrm{H}), 8.23(\mathrm{~d}, J=7.2 \mathrm{~Hz}$, $1 \mathrm{H}) ;{ }^{13} \mathrm{C}$ NMR $\left(\mathrm{CDCl}_{3}, 100 \mathrm{MHz}\right) \delta: 29.4,56.0,56.1$, $108.3,109.9,111.2,111.4,111.6,113.2,114.7,119.5$, $122.5,122.9,124.0,134.6,140.7,149.4,152.8,160.8$, 161.7, 176.6; IR (KBr) v: $1767(\mathrm{C}=\mathrm{O}), 1634(\mathrm{C}=\mathrm{O})$ $\mathrm{cm}^{-1}$. Anal. calcd for $\mathrm{C}_{21} \mathrm{H}_{17} \mathrm{NO}_{5}$ : C 69.41, H 4.72, N 3.85; found C 69.55, H 4.74, N 3.86 .

1-甲基-2-(2,4,5-三甲氧基苯基)-6-甲基吡喃并 [3,2-c]喹啉-4,5-二酮 (4h): 黄色针状晶体. m.p. $>300^{\circ} \mathrm{C}$; ${ }^{1} \mathrm{H}$ NMR $\left(\mathrm{CDCl}_{3}, 400 \mathrm{MHz}\right) \delta: 3.72\left(\mathrm{~s}, 3 \mathrm{H}, \mathrm{NCH}_{3}\right), 3.89$ $\left(\mathrm{s}, 3 \mathrm{H}, \mathrm{OCH}_{3}\right), 3.94\left(\mathrm{~s}, 3 \mathrm{H}, \mathrm{OCH}_{3}\right), 3.98\left(\mathrm{~s}, 3 \mathrm{H}, \mathrm{OCH}_{3}\right)$, $6.59(\mathrm{~s}, 1 \mathrm{H}), 7.23(\mathrm{~s}, 1 \mathrm{H}), 7.35(\mathrm{~s}, 1 \mathrm{H}), 7.37 \sim 7.43(\mathrm{~m}$, 2H), $7.73(\mathrm{t}, J=7.6 \mathrm{~Hz}, 1 \mathrm{H}), 8.18(\mathrm{~d}, J=8.0 \mathrm{~Hz}, 1 \mathrm{H}) ;{ }^{13} \mathrm{C}$ NMR $\left(\mathrm{CDCl}_{3}, 100 \mathrm{MHz}\right) \delta$ : 29.5, 56.2, 56.4, 56.7, 96.9, $109.9,110.5,111.2,113.2,113.3,114.9,122.5,122.7$, $123.9,134.3,140.8,143.1,153.4,154.5,159.6,161.8$, 176.8; IR (KBr) v: $1771(\mathrm{C}=\mathrm{O}), 1635(\mathrm{C}=\mathrm{O}) \mathrm{cm}^{-1}$. Anal. calcd for $\mathrm{C}_{22} \mathrm{H}_{19} \mathrm{NO}_{6}$ : C 67.17, H 4.87, N 3.56; found $\mathrm{C}$ $67.25, \mathrm{H} 4.88, \mathrm{~N} 3.55$.

1-甲基-2-(4-氯苯基)-6-甲基吡喃并[3,2-c]喹啉-4,5二酮 (4i): 黄色针状晶体. m.p. $>300{ }^{\circ} \mathrm{C} ;{ }^{1} \mathrm{H}$ NMR $\left(\mathrm{CDCl}_{3}, 400 \mathrm{MHz}\right) \delta: 3.72\left(\mathrm{~s}, 3 \mathrm{H}, \mathrm{NCH}_{3}\right), 6.86(\mathrm{~s}, 1 \mathrm{H})$, $7.36 \sim 7.43(\mathrm{~m}, 2 \mathrm{H}), 7.53(\mathrm{~d}, J=8.0 \mathrm{~Hz}, 2 \mathrm{H}), 7.74 \sim 7.77$ (m, 1H), $7.81(\mathrm{~d}, J=8.0 \mathrm{~Hz}, 2 \mathrm{H}), 8.24(\mathrm{~d}, J=7.8 \mathrm{~Hz}, 1 \mathrm{H})$; ${ }^{13} \mathrm{C}$ NMR $\left(\mathrm{CDCl}_{3}, 100 \mathrm{MHz}\right) \delta: 27.6,109.9,111.0,111.2$, $112.9,120.7,122.2,125.4,127.4,127.8,132.5,136.4$, 139.0, 156.5, 158.1, 160.1, 173.9; IR (KBr) $v: 1768(\mathrm{C}=$ O), $1637(\mathrm{C}=\mathrm{O}) \mathrm{cm}^{-1}$. Anal. calcd for $\mathrm{C}_{19} \mathrm{H}_{12} \mathrm{ClNO}_{3}$ : C 67.56, H 3.58, N 4.15; found C 67.68, H 3.60, N 4.16.

1-甲基-2-(2-氟苯基)-6-甲基吡喃并 [3,2-c]喹啉-4,5二酮 $(\mathbf{4 j})$ : 黄色针状晶体. m.p. $216 \sim 218{ }^{\circ} \mathrm{C} ;{ }^{1} \mathrm{H}$ NMR $\left(\mathrm{CDCl}_{3}, 400 \mathrm{MHz}\right) \delta: 3.71\left(\mathrm{~s}, 3 \mathrm{H}, \mathrm{NCH}_{3}\right), 6.98(\mathrm{~s}, 1 \mathrm{H})$, $7.23 \sim 7.340(\mathrm{~m}, 4 \mathrm{H}), 7.51 \sim 7.54(\mathrm{~m}, 1 \mathrm{H}), 7.73(\mathrm{dd}, J=$ 7.6, $8.0 \mathrm{~Hz}, 1 \mathrm{H}), 7.86(\mathrm{dd}, J=7.2,7.6 \mathrm{~Hz}, 1 \mathrm{H}), 8.21(\mathrm{~d}$, $J=8.0 \mathrm{~Hz}, 1 \mathrm{H}) ;{ }^{13} \mathrm{C} \mathrm{NMR}\left(\mathrm{CDCl}_{3}, 100 \mathrm{MHz}\right) \delta: 29.4$, $111.6,113.7,114.8,117.1,119.2,122.6,124.2,124.9$, $128.6,133.3,134.3,140.7,156.9,158.5,159.7,161.6$, 
162.1, 175.8. IR (KBr) v: $1764(\mathrm{C}=\mathrm{O}), 1644(\mathrm{C}=\mathrm{O})$ $\mathrm{cm}^{-1}$. Anal. calcd for $\mathrm{C}_{19} \mathrm{H}_{12} \mathrm{FNO}_{3}: \mathrm{C} 71.02, \mathrm{H} \mathrm{3.76,} \mathrm{N}$ 4.36; found C 71.17, H 3.75, N 4.37.

1-甲基-2-(呋喃-2-基)-6-甲基吡喃并[3,2-c]喹啉-4,5二酮 (4k): 黄色针状晶体. m.p. $>300{ }^{\circ} \mathrm{C} ;{ }^{1} \mathrm{H}$ NMR $\left(\mathrm{CDCl}_{3}, 400 \mathrm{MHz}\right) \delta: 3.71\left(\mathrm{~s}, 3 \mathrm{H}, \mathrm{NCH}_{3}\right), 6.63 \sim 6.64(\mathrm{~m}$, $1 \mathrm{H}), 6.78(\mathrm{~s}, 1 \mathrm{H}), 7.14 \sim 7.17(\mathrm{~m}, 1 \mathrm{H}), 7.33 \sim 7.41(\mathrm{~m}$, 2H), $7.64 \sim 7.65(\mathrm{~m}, 1 \mathrm{H}), 7.74(\mathrm{t}, J=7.6 \mathrm{~Hz}, 1 \mathrm{H}), 8.19$ (d, $J=7.6 \mathrm{~Hz}, 1 \mathrm{H}) ;{ }^{13} \mathrm{C} \mathrm{NMR}\left(\mathrm{CDCl}_{3}, 100 \mathrm{MHz}\right) \delta: 29.5$, $110.0,110.2,111.7,112.5,112.9,113.0,114.7,122.5$, 124.0, 134.3, 140.8, 145.1, 146.1, 153.0, 161.5, 175.4; IR $(\mathrm{KBr}) v: 1761(\mathrm{C}=\mathrm{O}), 1635(\mathrm{C}=\mathrm{O}) \mathrm{cm}^{-1}$. Anal. calcd for $\mathrm{C}_{17} \mathrm{H}_{11} \mathrm{NO}_{4}$ : C 69.62, H 3.78, N 4.78; found $\mathrm{C} 69.76, \mathrm{H}$ 3.79, N 4.77 .

1-甲基-2-(噻吩-2-基)-6-甲基吡喃并[3,2-c]喹啉-4,5二酮 (4I): 黄色针状晶体. m.p. $>300{ }^{\circ} \mathrm{C} ;{ }^{1} \mathrm{H}$ NMR $\left(\mathrm{CDCl}_{3}, 400 \mathrm{MHz}\right) \delta: 3.71\left(\mathrm{~s}, 3 \mathrm{H}, \mathrm{NCH}_{3}\right), 6.76(\mathrm{~s}, 1 \mathrm{H})$, $7.20 \sim 7.23(\mathrm{~m}, 1 \mathrm{H}), 7.34 \sim 7.40(\mathrm{~m}, 2 \mathrm{H}), 7.59(\mathrm{~d}, J=7.6$ $\mathrm{Hz}, 1 \mathrm{H}), 7.68 \sim 7.75(\mathrm{~m}, 2 \mathrm{H}), 8.22(\mathrm{~d}, J=8.0 \mathrm{~Hz}, 1 \mathrm{H}) ;{ }^{13} \mathrm{C}$ NMR $\left(\mathrm{CDCl}_{3}, 100 \mathrm{MHz}\right) \delta$ : 27.5, 109.1, 109.8, 111.1, $112.8,120.7,122.2,126.3,126.9,128.5,131.9,132.4$, $138.9,154.8,156.5,159.6,173.6$; IR $(\mathrm{KBr}) v: 1764(\mathrm{C}=$ O), $1637(\mathrm{C}=\mathrm{O}) \mathrm{cm}^{-1}$. Anal. calcd for $\mathrm{C}_{17} \mathrm{H}_{11} \mathrm{NO}_{3} \mathrm{~S}: \mathrm{C}$ 66.01, H 3.58, N 4.53; found C 66.12, H 3.59, N 4.53.

辅助材料(Supporting Information) 合成产物的 ${ }^{1} \mathrm{H}$ $\mathrm{NMR}$ 和 ${ }^{13} \mathrm{C} \mathrm{NMR}$ 图谱. 这些材料可以免费从本刊网站 (http://sioc-journal.cn/)上下载.

\section{References}

[1] (a) Chen, J. J.; Chen, P. H.; Liao, C. H.; Huang S. Y.; Chen, I. S. J. Nat. Prod. 2007, 70, 1444

(b) Hanawa, F.; Fokialakis N.; Skaltsounis, A. L. Planta Med. 2004, 70,531 .

(c) Madkour, H. M. F.; Mahmoud, M. R.; Sakr, A. M.; Habasy, M. M. Sci. Pharm. 2001, 69, 33.

[2] Chen, I. S.; Wu, S. J.; Tsai, I. L.; Wu, T. S.; Pezzuto, J. M.; Lu, M. C.; Chai, H.; Suh N.; Teng, C. M. J. Nat. Prod. 1994, 57, 1206.

[3] (a) Barr, S. A.; Neville, C. F.; Grundon, M. F.; Boyd, D. R.; Malone, J. F.; Evans, T. A. J. Chem. Soc. Perkin Trans. 1 1995, 445. (b) Ngadjui, B. T.; Ayafor, J. F.; Bilon, A. E. N.; Sondengam, B. L.; Connolly J. D.; Rycroft, D. S. Tetrahedron, 1992, 48, 8711. (c) Grundon, M. F. Tetrahedron, 1978, 34, 143.

[4] Ghorab, M. M.; Heiba, H. I.; Amin, N. E. Pharmazie 1999, 54, 226.

[5] Tavares, L. C.; Johann, S.; Alves, T. M. A.; Guerra, J. C.; Souza-Fagundes, E. M.; Cisalpino, P. Silva.; Bortoluzzi, A. J.; Caramori, G. F.; Piccoli. R. M.; Braibante, H. T. S.; Braibante, M. E. F.; Pizzolatti, M. G. Eur. J. Med. Chem. 2011, 46, 4448.

[6] Guo, R. H.; Zhang, Q.; Ma, Y. B.; Luo, J.; Geng, C. A.; Wang, L. J.; Zhang, X. M.; Zhou, J.; Jiang, Z. Y.; Chen. J. J. Eur. J. Med. Chem.
2011, 46, 307.

[7] Curreli, F.; Zhang, H.; Zhang, X.; Pyatkin, I.; Victor, Z.; Altieri, A.; Debnath, A. K. Bioorg. Med. Chem. 2011, 19, 77.

[8] (a) Rahman, M. M.; Gray, A. I.; Khondkar, P.; Sarker, S. D. Pharm. Biol. 2008, 46, 356.

(b) Meragelman, T. L.; Tucker, K. D.; McClord, T. G.; Cardel-lina, J. H.; Shoemker, R. H. J. Nat. Prod. 2005, 68, 1790.

[9] Hirpara, K. V.; Aggarwal, P.; Mukherjee, A. J.; Joshi, N.; Burman, A. C. Curr. Med. Chem. 2009, 9, 138.

[10] (a) Mao, J.; Hua, Q.; Xie, G.; Guo, J.; Yao, Z.; Shi, D.; Ji, S. Adv. Synth. Catal. 2009, 351, 635.

(b) Naik, M. M.; Kamat, D. P.; Tilve, S. G.; Kamat, V. P. Tetrahedron 2014, 70, 5221.

[11] (a) Parvatkar, P. T.; Parameswaran, P. S.; Tilve, S. G. Chem. Eur. J. 2012, 18, 5460.

(b) Shen, S. S.; Xu, X. P.; Ji, S. J. Chin. J. Org. Chem. 2009, 29, 806 (in Chinese).

(沈舒苏, 徐小平, 纪顺俊, 有机化学, 2009, 29, 806.)

[12] (a) Yin, G.; Fan, L.; Ren, T.; Zheng, C.; Tao, Q.; Wu, A.; She, N. Org. Biomol. Chem. 2012, 10, 8877.

(b) Ahmed, N.; Babu, B. V. Synth. Commun. 2013, 43, 3044.

[13] (a) Panarese, J. D.; Waters, S. P. Org. Biomol. Chem. 2013, 11, 3428.

(b) Wu, X.; Gao, Q.; Liu, S.; Wu, A. Org. Lett. 2014, 16, 2888.

[14] (a) Meng, D. J.; Chen, R. X.; Liu, L. T.; Wang, T.; Liu, X. M.; Zhao, W. X. Chin. J. Org. Chem. 2015, 35, 2108 (in Chinese).

(孟团结, 陈荣祥, 刘澜涛, 王 涛, 刘新明, 赵文献, 有机化学, 2015, 35, 2108.)

(b) Luo, Q.; Xie, Y. X.; Chen, C. Y.; Yan, S. Y.; Den, W. Y. Chin. J. Org. Chem. 2014, 34, 2537 (in Chinese).

(罗倩, 谢永新，陈朝阳，问世友，邓文叶，有机化学，2014，34， 2537.)

(c) Hu, F.; Gao, W. C.; Chang, H. H.; Li, X.; Wei, W. L. Chin. J. Org. Chem. 2015, 35, 1848 (in Chinese).

(胡飞, 高文超, 常宏宏, 李兴, 魏文珑, 有机化学, 2015, 35, 1848.)

(d) Wang, D. Y.; Zhang, R. X.; Lin, S.; Deng, R. H.; Yan, Z. H. Chin. J. Org. Chem. 2016, 36, 2757 (in Chinese).

(王丁意, 张荣兴, 林森, 邓瑞红, 严兆华, 有机化学, 2016, 36, 2757.)

[15] (a) Wang, D. L.; Wu, D.; Zhao, W.; Wang, Y. Y.; Wu, J. Y. Chin. Chem. Lett. 2015, 26, 251.

(b) Wang, D. L.; Zhou, T.; Xing, J. J.; Qiang, J. H.; Liu, L. Heterocycles, 2016, 92, 733 .

(c) Wang, D. L.; Shi, X. C.; Wang, Y. Y.; Ma, J. Chin. Chem. Lett. 2016, 27, 261.

[16] (a) Ye, J. H.; Wu, J.; Huang, N. F.; Zhang, W. C.; Yu, S. Y.; Qin, Z. C.; Wang, Z. Y.; Shang, Y. J. Chin. J. Org. Chem. 2010, 30, 1548 (in Chinese).

(叶家海, 吴 俊, 黄年峰, 张文超, 余述燕, 秦志春, 王智勇, 商 永嘉, 有机化学, 2010, 30, 1548.)

(b) Munawar, M. A.; Azad, M.; Siddiqui, H. L.; Nasim, F. H. J. Chin. Chem. Soc. 2008, 55, 394.

(c) Azad, M.; Munawar, M. A.; Siddiqui, H. L. J. Applied Sci. 2007, 7,2485 .

[17] (a) Wang, D. L.; Yang, F. F.; Liu, Z.; Dong, Z.; Zhao, W. Chin. J. Org. Chem. 2014, 34, 204 (in Chinese).

(王道林, 杨菲菲, 刘忠, 董哲, 赵伟, 有机化学, 2014, 34, 204.)

(b) Gao, W. T.; Cheng, X. P.; Li, Y. Chin. J. Org. Chem. 2010, 30, 456 (in Chinese)

(高文涛，程秀萍，李阳，有机化学，2010, 30, 456.) 
[18] (a) Menezes, M. J.; Manjrekar, S.; Pai, V.; Patre, R. E.; Tilve, S. G. Indian J. Chem., Sect. B 2009, 48, 1311.

(b) Gao, W.; Li, Y.; Zhang, H.; Chang, M. Q.; Imafuku, K. J. Heterocyel. Chem. 2009, 46, 1107.
[19] Santosusso, T. M.; Swern, D. J. Org. Chem. 1975, 40, 2764.

[20] Chen, H.; Li, J.; Chen, G. L.; Zhou, X.; Lu, X. H. Chem. Reag. 2010, 32, 80 (in Chinese).

(陈浩，李杰，陈国良，周勋，陆秀宏，化学试剂，2010, 32, 80.)

(Li, L.; Fan, Y.) 